\title{
La polémica entre bonaventura cavalieri y Paul Guldin o jesuatos contra jesuitas sobre los indivisibles
}

\author{
Vernor Arguedas \\ vernor.arguedas@ucr.ac.cr \\ Escuela de Matemática \\ Universidad de Costa Rica
}

Resumen. Se analiza la polémica entre Cavalieri y Guldin sobre el concepto de de indivisible.

Palabras clave: Cavalieri, Guldin, orden de los jesuatos, orden de los jesuitas, guerra de los treinta años, principio de Cavalieri.

Abstract. It is analyzed the polemic between Cavalieri and Guldin on the concept of indivisible.

KeyWords: Cavalieri, Guldin, Jesuati order,Jesuit order, thirty year war, Cavalieri's principle.

El parto del cálculo o análisis matemático no fue fácil. Variar las técnicas de demostración no fue un proceso sencillo.

Varias veces he afirmado que fue gracias a Euler que este campo fue reconocido en su importancia, en sus métodos y sobre todo en sus aplicaciones. Hay varias polémicas muy interesantes: la del obispo Berkeley contra la escuela de Newton y la que vamos a analizar en este artículo entre Cavalieri y Guldin. Cavalieri (1598-1647, jesuato) y Guldin (1577-1643, jesuita)viven en el periodo de la guerra de los treinta años (1618-1648) que posiblemente sea el primer gran conflicto global . Un resumen sobre esta guerra devastadora en Europa se puede leer en: http://es.wikipedia.org/wiki/Guerra_de_ los_Treinta_A\%C3\%B1os.

Los jesuatos fueron los miembros de una orden religiosa poco conocida (1367-1668, http: //es . wikipedia. org/wiki/Jesuatos). 
El Papa Urbano V, en el año 1367, aprobó esta congregación con la condición de que establecieran monasterios propios. Su hábito estaba formado por una túnica blanca, con capucha cuadrada y un manto gris-marrón. Era una orden menor, hasta que en 1606 se les permitió tener uno o dos sacerdotes por convento. Fue suprimida por el Papa. Clemente IX en 1668.

La orden de los jesuitas es muy conocida, algunos datos se pueden leer en http://es. wikipedia. org/wiki/Compa\%C3\%B1\%C3\%ADa_de_Jes\%C3\%BAs; el Papa Francisco es el primer jesuita en acceder a este puesto.

Algunos breves aspectos biográficos de Guldin y Cavalieri se encuentran en: http://es. wikipedia. org/wiki/Paul_Guldin y http://es.wikipedia.org/wiki/Bonaventura_Cavalieri

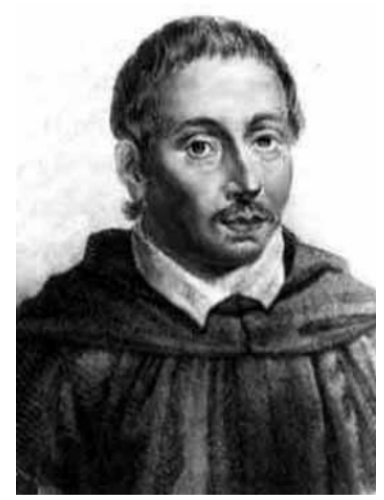

Figura 1.1: Cavalieri

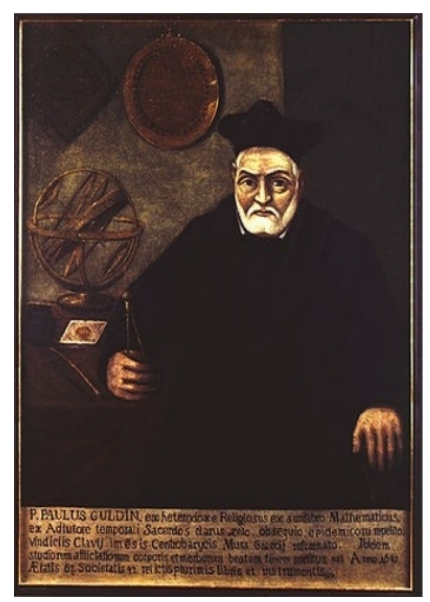

Figura 1.2: Guldin

Cavalieri fue discípulo de Galileo, trabajó y publicó varios libros de matemática entre otros: "Tratado de las secciones cónicas"; "Directorium generale uranometricum"; y los fundamentales. "Geometria continuorum indivisibilibus nova quadam ratione promota libri sex", en 1635 en Bologna, y sus "Exercitationes geometricae sex" en 1647. De la "Geometria indivisibilus" circularon varias versiones desde unos años antes de la publicación oficial, en ésta, su obra principal, se establece un nuevo principio.

El principio de la nueva geometría consistía en sustituir la figura plana por los agregados de todas las líneas y las figuras sólidas por los agregados de los infinitos planos que en ellos se contienen. Para mostrar la validez de sus demostraciones, Cavalieri aplicó su método a demostraciones ya conocidas obteniendo los mismos resultados. Es este uso del concepto infinito lo que critica Guldin, para quien esta metodología no es constructiva en el sentido geométrico de Euclides, ni respeta el pensamiento aristotélico dominante, en esa época, en la orden de los jesuitas y en muchos sectores de la iglesia Católica. La controversia científica pronto se transformó en una violenta polémica, sobre todo tras la muerte, acaecida en 1643, del matemático jesuíta Paul Guldin, quien estaba radicalmente en contra del método de Cavalieri pues rechazaba que los agregados de infinitas líneas, o de infinitos planos, pudieran compararse entre sí. «Entre infinito e infinito -observaba Guldin- no hay relación». Pero replica Cavalieri-los infinitos puntos de un segmento, por ejemplo, no son infinitos in ratione totius, es decir, como lo es un todo infinito: a ellos es siempre posible quitarles o añadirles otros puntos. 
La discusión es profunda y la argumentación de Guldin se encuentra en su obra más importante Centrobaryca (1635-1641), esta obra consta de 4 volúmenes y en los últimos se refiere al principio de los indivisibles.

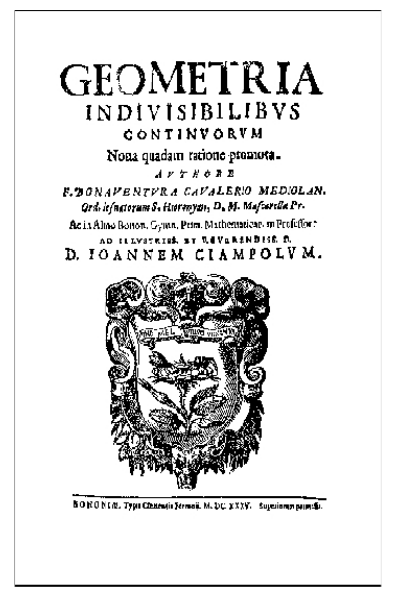

Figura 1.3: Geometria indivisibilus

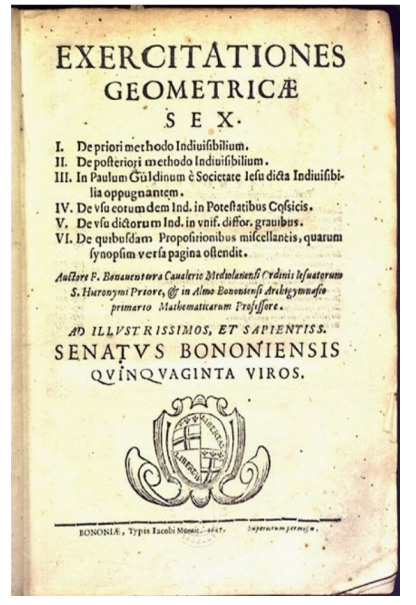

Figura 1.4: Exercitationes geometricae sex

Algunas imágenes de la obra de Cavalieri nos aclaran cómo visualizaba este autor su principio, el cual aplicaba a figuras planas y sólidas.

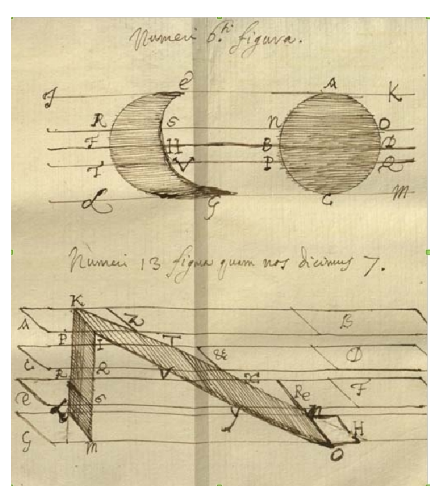

Figura 1.5

En Italia los indivisibles geométricos encuentran un defensor en Evangelista Torricelli (1608-1647), quien introduce la noción de indivisibles curvos en sus demostraciones.

Como se sabe, Torricelli es también el autor del experimento barométrico llevado a cabo en Florencia en 1644. La aparición de la región aparentemente vacía en el tubo de vidrio que contiene el mercurio viene a reavivar la polémica sobre la posible existencia del vacío que los aristotélicos negaban. En efecto, el vacío macroscópico sugiere la existencia del vacío microscópico y, por tanto, de los átomos.

La aversión de los jesuitas hacia la doctrina atomista está probablemente en el origen del silencio casi absoluto de Torricelli sobre los resultados obtenidos por él: el asunto sólo se trata en dos cartas, dirigidas a Michelangelo Ricci poco días después de que se llevara a cabo el experimento. Torricelli, por el contrario, le da una amplia difusión a sus trabajos matemáticos, en los que los indivisibles ocupan un 
puesto de primerísimo orden. Se diría que el debate en torno al atomismo geométrico se les deja sólo a los matemáticos, en tanto que aumenta el interés de los filósofos y de los teólogos por el atomismo físico y por los experimentos acerca del vacío.

En un artículo relativamente reciente, de 1991, Howard Eves. https://www.maa.org/sites/default/ files/pdf/upload_library/22/Polya/07468342.di020746.02p00037.pdf encuentra estos dos bellísimos resultados. El principio de Cavalieri (vea la figura anterior, según el dibujo original de Cavalieri) se formula de la siguiente manera:

1. Si dos figuras planas están contenidas entre un par de rectas paralelas y si las longitudes de los dos segmentos cortados por cualquier paralela, paralela a estas, son iguales, entonces las dos figuras tienen la misma área.

2. Si dos sólidos están contenidos entre dos planos paralelos y si las áreas de dos secciones de estos sólidos cortadas por un plano paralelo a estos planos son iguales, entonces los dos sólidos tienen el mismo volumen.

Teorema 1.1

Si dos triángulos tienen la misma área son congruentes Cavalieri ( es decir existen las rectas paralelas que cumplen el punto 1 del principio de Cavalieri).

La figura básica es la siguiente:

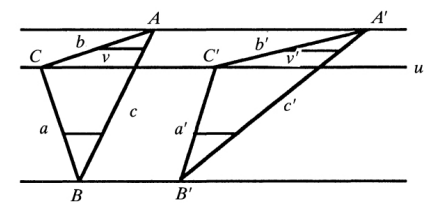

Figura 1.6

Teorema 1.2

Dada una esfera cualquiera existe un tetraedro tal que la esfera y el tetraedro son Cavalieri congruentes( es decir existen dos planos paralelos según el punto 2 del principio de Cavalieri).

La figura básica es la siguiente:

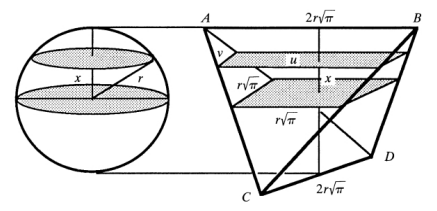

Figura 1.7 
Las figuras son del artículo del profesor Eves. El profesor Eves ganó el premio Polya por este artículo en 1992. http://en.wikipedia.org/wiki/George_P\%C3\%B3lya_Award

La argumentación de Guldin obligó a los matemáticos de los siglos siguientes a precisar los conceptos. Cavalieri no estaba en condiciones de explicar su método pues le faltaba el lenguaje matemático necesario. La teoría de la medida nace varios siglos después.

La contención de Guldin en su argumentación contra Cavalieri, dejando sólo en el plano matemático su disputa, es un misterio que huele a miedo a la poderosa inquisición. Después de todo él era un judío converso y el juicio a Galileo, que comenzó en 1633, recordaba que toda crítica o alejamiento de la ortodoxia podía conducir a la Sagrada Inquisición.

\section{Bibliografía}

[1] "La guerra de los treinta años" http://es.wikipedia.org/wiki/Guerra_de_los_Treinta_A\%C3\% B1os

[2] "Orden de los Jesuatos" http://es.wikipedia.org/wiki/Jesuatos

[3] "Orden de los Jesuitas" http://es.wikipedia.org/wiki/Compa\%C3\%B1\%C3\%ADa_de_Jes\%C3\%BAs

[4] "Biografía Paul Guldin" http://es.wikipedia.org/wiki/Paul_Guldin

[5] "Biografía Cavalieri" http://es.wikipedia.org/wiki/Bonaventura_Cavalieri

[6] Howard Eves. "Two Surprising Theorems on Cavalieri Congruence" (1991). https://www. maa.org/ sites/default/files/pdf/upload_library/22/Polya/07468342.di020746.02p00037.pdf

[7] "Premios George Polya" http://en.wikipedia.org/wiki/George_P\%C3\%B3lya_Award 